\title{
Pulmonary function in children after neonatal meconium aspiration syndrome
}

\author{
P I MACFARLANE AND D P HEAF \\ Respiratory Unit, Royal Liverpool Children's Hospital Alder Hey, Liverpool
}

SUMmARY Eighteen children aged 6 to 11 years old who fulfilled criteria for the diagnosis of neonatal meconium aspiration syndrome were investigated to find out the prevalence of previous and current respiratory symptoms, and abnormal pulmonary function tests, chest radiographs, and ventilation scans. Eleven of the 18 had no respiratory symptoms and eight of these had normal pulmonary function on testing. Two had mild limitation of expiratory airflow that did not respond to treatment with bronchodilators, and one had exercise induced bronchospasm that responded to treatment with bronchodilators. Seven of the 18 had recurrent cough and wheezing compatible with a diagnosis of asthma, and five of these had appreciable exercise induced bronchospasm that responded to treatment with bronchodilators. None of the children with symptoms had a personal or family history of atopy or had had acute bronchiolitis. All chest radiographs were normal.

We found a much higher prevalence of asthmatic symptoms and abnormal bronchial reactivity among survivors of neonatal meconium aspiration syndrome than in the general childhood population. Aspiration of meconium may have long term consequences for the developing respiratory tract and is associated with abnormal respiratory function in later childhood.

Neonatal meconium aspiration results in respiratory distress of variable severity that is characterised by obstruction of airways, trapping of gas, inequality of ventilation and perfusion and a tendency to persistent fetal circulation. About $10 \%$ of babies are born through amniotic liquor stained with meconium, and of these $20 \%$ develop clinical illness. ${ }^{\prime}$ Mortality is high, but among survivors the signs of respiratory distress seem to resolve completely. There has, however, been no study of the respiratory status of these children in later life. This is relevant because it is known that there are many respiratory illnesses in early life, both non-infectious and infectious, which appear to resolve but which are associated with impaired respiratory function in later years. ${ }^{2}$ There is also speculation that various insults to the developing respiratory tract in childhood may be determinants of chronic adult respiratory disease. ${ }^{3}$

The aim of this study was to investigate respiratory symptoms and function after five years in children who had had severe respiratory distress caused by the neonatal meconium aspiration syndrome.

\section{Methods}

DIAGNOSTIC CRITERIA

Case records of infants born between 1975 and 1980 and admitted to three neonatal units and one regional paediatric intensive care unit in Liverpool were examined to find those with a diagnosis of neonatal meconium aspiration syndrome. Patients were accepted into the study if all the following criteria were met: (i) amniotic liquor stained with meconium was apparent before delivery; (ii) a paediatrician was present at delivery who performed direct laryngoscopy and retrieved meconium from below the vocal cords by endotracheal suction; (iii) respiratory distress was apparent immediately after birth; (iv) inspired oxygen of greater than $40 \%$ was required more than 48 hours; (v) there were radiological features of meconium aspiration syndrome; and (vi) other causes of respiratory distress - for example, pneumonia, lung malformation, and respiratory distress syndrome-had been excluded.

Details of all those who fulfilled these criteria 
were recorded, and the parents were contacted by letter inviting them to allow the child to take part in the study, and to attend the respiratory function laboratory for further evaluation.

\section{RESPIRATORY SYMPTOMS}

Parents and children who attended for further study completed a questionnaire about respiratory symptoms based on selected questions from the American Thoracic Society's Epidemiology Standardization Project. ${ }^{4}$ This included questions about whether the child had recurrent coughs, phlegm, or wheezing; whether these symptoms were associated with upper respiratory tract infections or exercise; whether asthma had been diagnosed, and what drugs the child was taking. There were also questions about past respiratory illnesses (measles, bronchiolitis, pneumonia, pertussis, and croup), and about any history of atopy in parents or siblings (asthma, hayfever, or eczema). There were supplementary questions about the number of episodes of wheezing serious enough to cause shortness of breath in the previous 12 months, other health problems, and schooling. No attempt was made to assess the possible effects of environmental pollutants.

\section{PULMONARY FUNCTION TESTING}

All pulmonary function tests were performed in the morning in the summer months June to September, and no child had had an upper respiratory tract infection within four weeks of testing. Functional residual capacity was measured by the helium dilution method, ${ }^{5}$ using a PK Morgan multibreath closed circuit apparatus. After appropriate instruction and practise children performed maximal forced expirations into a turbine spirometer (Microloop, Micro Medical Ltd) linked to a microcomputer with visual display of the expiratory flow volume curve. The best of three forced expirations were used to record flow at $50 \%$ and $75 \%$ of forced vital capacity and the average flow between $25 \%$ and $75 \%$ of vital capacity. In addition, the volume expired in the first second of forced vital capacity and the forced vital capacity itself, were recorded from maximal expirations into a hand held electronic digital spirometer (pocket spirometer, Micro Medical Ltd). Peak expiratory flow rate was measured by a Wright peak flow meter.

As a test of exercise induced bronchospasm and response to treatment with bronchdilators, each child then undertook a free running exercise test lasting six minutes with measurements of peak expiratory flow rate at 0,3 , and 5 minutes of exercise, and at 3, 9, and 15 minutes after the end of the exercise. Salbutamol $200 \mu \mathrm{g}$ was then inhaled by aerosol through a spacer device (Volumatic) and further peak expiratory measurements made five and 10 minutes later.

\section{LUNG IMAGING}

A plain posteroanterior chest radiograph was taken and each patient underwent $81 \mathrm{~m}$ krypton ventilation scanning by steady state continuous tidal breathing.

Approval for the study was given by the hospital ethics committee.

\section{ANALYSIS OF DATA}

Measurements of peak expiratory flow from the exercise challenge were used to calculate the exercise induced bronchospasm index. This was calculated by the following formula: pre-exercise peak expiratory flow rate - minimum peak expiratory flow rate after exercise $\div$ pre-exercise peak expiratory flow rate $\times 100 \%$. The $\%$ response to bronchodilators was calculated from: maximum post-bronchodilator peak expiratory flow rate peak expiratory flow rate at 15 minutes postexercise $\div$ maximum post-bronchodilator peak expiratory flow rate $\times 100 \%$.

Pulmonary function test values were expressed as $\%$ predicted for height and sex. ${ }^{67}$ Individual test values were considered abnormal if they were more than two standard deviations below the mean for composite reference values, ${ }^{6}$ or below the normal (lower) 95th centile for maximal expiratory flow volume curve reference values. ${ }^{7}$ For the exercise test, an exercise induced bronchospasm index of greater than $10 \%,{ }^{8}$ and a bronchodilator response of greater than $15 \%$ were considered abnormal.

Radiographs and $81 \mathrm{~m}$ krypton ventilation scans were reported independently by a radiologist not taking part in the study.

\section{Results}

During the six year period 72 neonates were diagnosed in their medical records as having 'meconium aspiration syndrome'. Only 34 of these, however, fulfilled the strict criteria outlined for this study.

Six of the 34 died; all had received mechanical ventilation and they all died from birth asphyxia or severe meconium aspiration, or both. Of the remaining 28 children, 10 families had moved from the address given at the time of the child's birth, but four of these were traced and completed the questionnaire by post or telephone. Thus 18 children attended for testing of pulmonary function. Neonatal details of those children who did not attend for pulmonary function testing $(n=10)$ did 
not differ significantly from those of the ones who were tested $(n=18)$. The table summarises the neonatal details of the 18 children studied. Two babies had received full mechanical ventilation and four others had developed pneumomediastinum and pneumothrax.

At follow up the mean age was 7.8 years (range 5.7-11.2) and all except one was of normal height (mean height standard deviation score $=-0 \cdot 12$, range $+1 \cdot 24$ to $-3 \cdot 1$ )

\section{RESPIRATORY SYMPTOMS}

Eleven children were completely healthy without any current or previous respiratory symptoms. Seven children had recurrent coughs and wheezing associated with upper respiratory tract infections of

Table Neonatal details of 18 children followed up five years after having had meconium aspiration syndrome

\begin{tabular}{|c|c|c|}
\hline & Mean & Range \\
\hline Gestation (weeks) & $40 \cdot 3$ & $38-42$ \\
\hline Birthweight (g) & 3550 & $2620-4250$ \\
\hline $\begin{array}{l}\text { Maximum fractional inspired oxygen } \\
\text { concentration }\end{array}$ & 0.5 & $0.4-1$ \\
\hline Duration of oxygen requirement (hours) & 90 & $48-192$ \\
\hline Apgar score: & & \\
\hline at 1 minute & $4 \cdot 5$ & $1-8$ \\
\hline at 5 minutes & $7 \cdot 2$ & $1 \cdot 10$ \\
\hline at 10 minutes & $8 \cdot 1$ & $5-9$ \\
\hline
\end{tabular}

whom six also experienced coughs and wheezing at times other than during infections or during exercise. Five of these seven had had more than two episodes of wheezing serious enough to cause shortness of breath in the previous 12 months. These seven symptomatic children had no family history of atopy and none had had acute bronchiolitis; one had had croup. In this group two families were aware that their child had 'recurrent bronchitis' and two had been diagnosed as having asthma and treated by their family doctor.

Of the 11 asymptomatic children, four had a family history of atopy, two had had croup, and one had had acute bronchiolitis.

Thus, seven of 18 survivors of meconium aspiration syndrome had recurrent symptoms compatible with asthma, and this was not explained by a family history of atopy, or a history of respiratory illness after the neonatal period.

\section{PULMONARY FUNCTION TESTS}

The results of each child's tests are shown in figs 1 , 2 , and 3 , and compared with the normal reference ranges. 67

\section{SYMPTOMATIC GROUP}

Among the seven symptomatic children, two had normal pulmonary function tests both at rest and after exercise. The remaining five all had appreciable bronchoconstriction when they exercised and
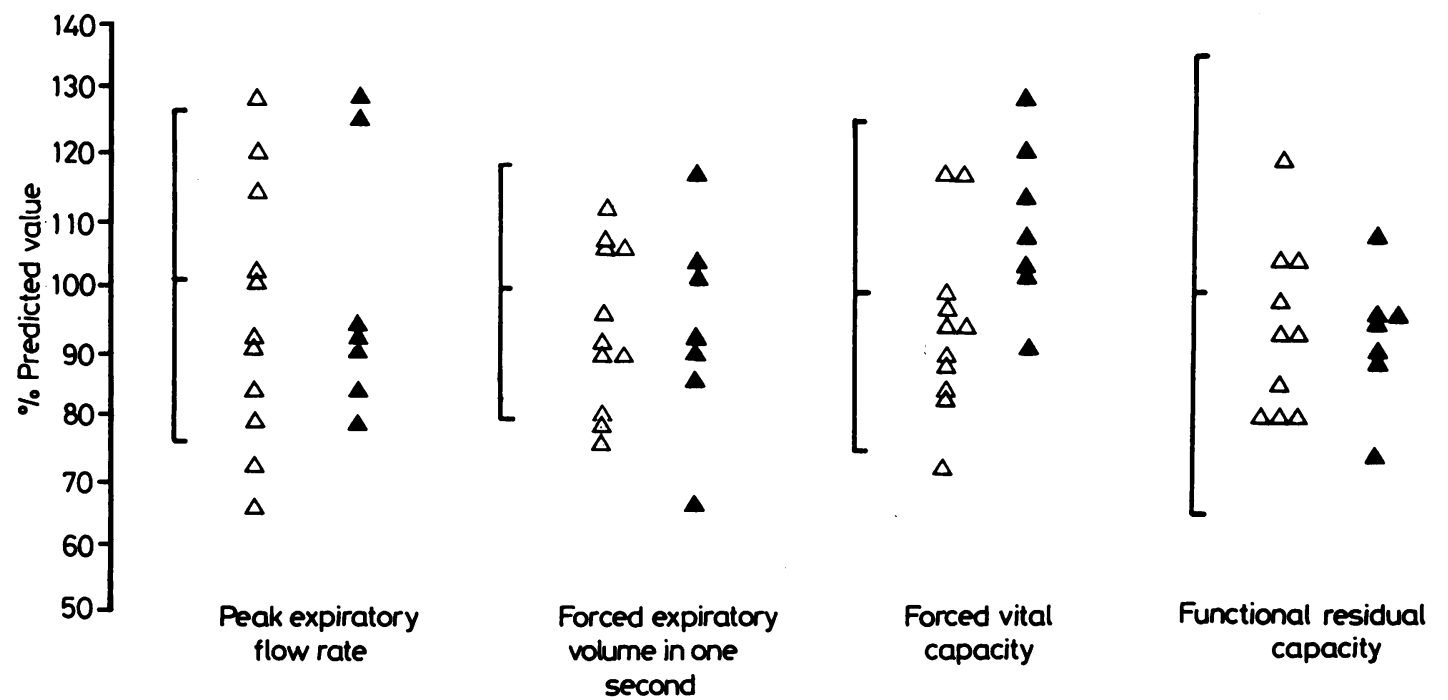

Fig 1 Peak expiratory flow rate, forced expiratory volume in one second, forced vital capacity, and functional residual capacity in 18 children who had had neonatal meconium aspiration syndrome, shown as \% predicted; normal ranges for each test indicated by bars. ${ }^{\circ} \Delta=$ symptomatic children and $\Delta=$ asymptomatic children. 


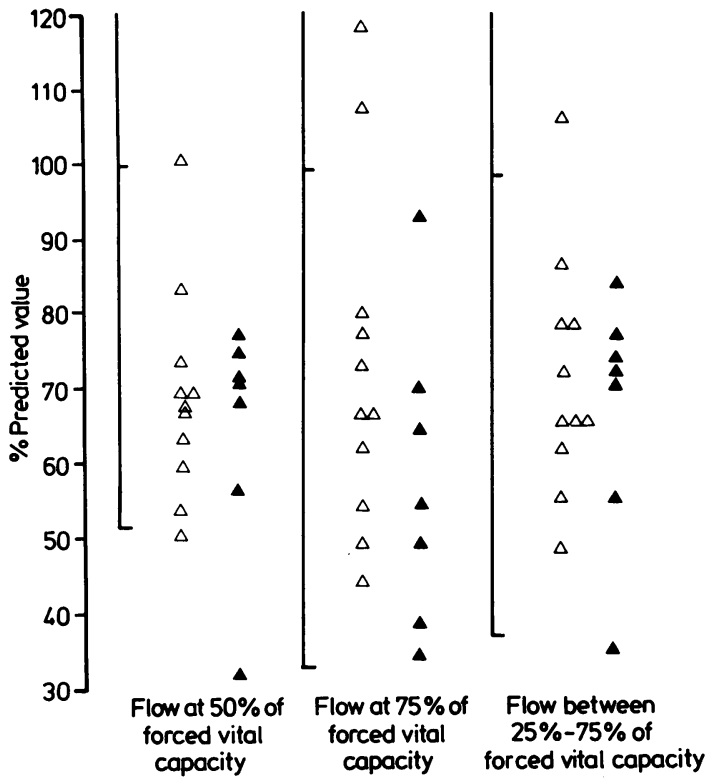

Fig 2 Flow at $50 \%$ forced vital capacity, flow at $75 \%$ forced vital capacity, and flow between $25 \%$ and $75 \%$ of forced vital capacity in 18 children who had had neonatal meconium aspiration syndrome, shown as \% predicted; normal ranges and lower 95 th centile are indicated by bars. $\mathbf{\Delta}=$ symptomatic children and $\triangle=$ asymptomatic children.

all responded to treatment with bronchodilators. One child who was known to be asthmatic had low forced expiratory volume at one second, low flow at $50 \%$ of forced vital capacity, and low flow between 25 and $75 \%$ of vital capacity at rest. This patient and one other who responded to treatment with bronchodilators had abnormal ventilation scans that showed moderate generalised decreased air entry to the left lung. All the chest radiographs were normal.

\section{ASYMPTOMATIC GROUP}

Among the 11 asymptomatic children, eight had normal pulmonary function both at rest and after exercise. One child had significant exercise induced bronchospasm $(20 \%$ fall $)$, and responded to treatment with bronchodilators ( $16 \%$ rise). The remaining two children had low peak expiratory flow rate, low forced expiratory volume at one second, and low flow at $50 \%$ of vital capacity that did not vary with exercise or respond to inhaled bronchodilators.

All chest radiographs and ventilation scans were normal in this group.

Ten of the 18 survivors $(56 \%)$ of meconium aspiration syndrome had normal pulmonary function tests. Six of the $18(33 \%)$ had abnormal bronchial reactivity.

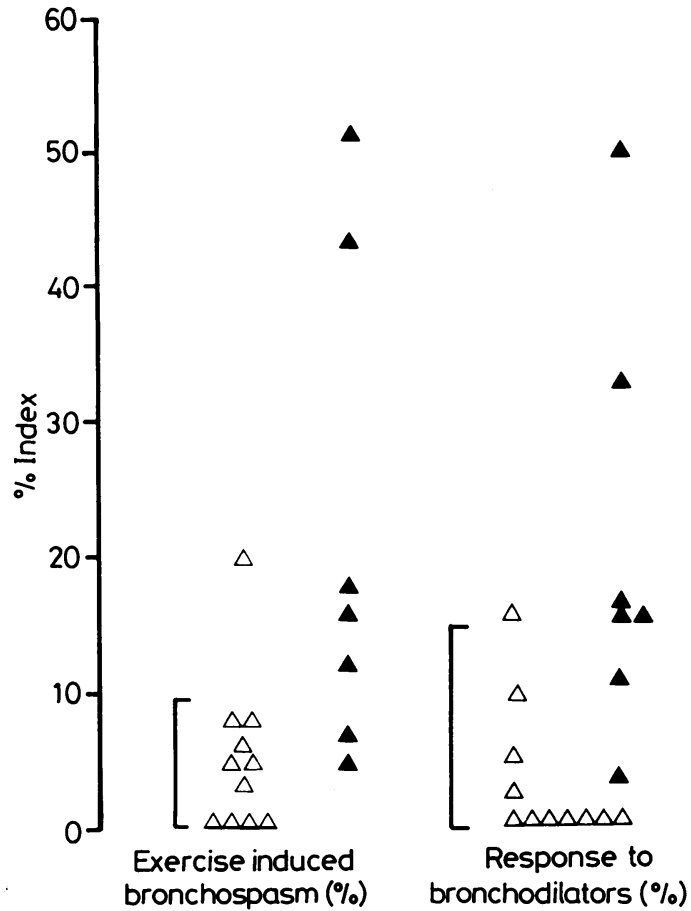

Fig 3 Exercise induced bronchospasm index (\%) and response to treatment with bronchodilators (\%) in 18 children who had had neonatal meconium aspiration syndrome. Ranges and upper limit of normal are indicated by bars. ${ }^{8} \boldsymbol{\Lambda}=$ symptomatic children and $\triangle$ asymptomatic children.

The severity of the neonatal meconium aspiration syndrome did not seem to correlate with the presence of symptoms or abnormal pulmonary function. The two children who received mechanical ventilation for meconium aspiration syndrome were symptomatic, but neither had abnormalities of pulmonary function at rest or after exercise.

\section{Discussion}

We found a high prevalence of asthmatic symptoms $(39 \%)$ and abnormal bronchial reactivity to exercise $(33 \%)$ among survivors of neonatal meconium aspiration syndrome. This is much higher than the estimated prevalence of $10-12 \%$ in the general childhood population at this age. ${ }^{910}$ These children were not atopic or from atopic families, and with one exception (croup) had not suffered any other respiratory insult known to be associated with bronchial reactivity in later childhood. The failure to diagnose asthma among the symptomatic children in our study was comparable with that found in larger community studies. ${ }^{910}$ 
By our inclusion criteria for diagnosing meconium aspiration syndrome we probably excluded the milder forms of the disease, hence the findings of this study apply only to moderate and severe meconium aspiration syndrome. We were only able to test fully 18 of the 28 surviving children initially identified as having serious neonatal meconium aspiration syndrome. The effect of selection bias on our findings must therefore be considered. Six families could not be traced because they had moved from our region and can therefore legitimately be excluded from analysis. Four children declined to attend for pulmonary function testing but answered the respiratory symptom questionnaire; three were asymptomatic but one had 'recurrent bronchitis' with symptoms of recurrent cough, wheezing and breathlessness, and used bronchodilators occasionally. Thus it would not seem that there was selection bias. Furthermore, even if all children had been fully evaluated the group would still contain larger numbers of children with asthmatic symptoms and bronchial reactivity than the general population; hence the main conclusion of the study would be unchanged.

Among the 11 asymptomatic children there were two who had evidence of mild fixed obstructive airways disease on pulmonary function testing, with limitation of expiratory airflow at various lung volumes. All the other children had test values within normal limits, but tended towards the lower limit of normal (fig 2).

Our findings of abnormal bronchial reactivity to exercise and mild expiratory airflow limitation makes neonatal meconium aspiration syndrome another factor in the wide range of insults to the developing respiratory tract that may cause abnormalities of pulmonary function in later life. Aspiration of other foreign material (such as hydrocarbons $^{11}$ or fresh water ${ }^{12}$ ) is associated with later abnormalities of pulmonary function, especially abnormal bronchial reactivity, long after symptoms have resolved.

It seems likely that the developing respiratory tract is vulnerable to damage by many insults, and the non-specific response is abnormal bronchial reactivity and limitation of airflow. ${ }^{13}$

We thank Mrs Dorothy Bolger and Mrs Sandra Longworth for typing the manuscript and Mr Ken Walters for providing the illustrations.

\section{References}

' Gregory G. Gooding CA. Phibbs RM. Tooley WH. Meconium aspiration in infants--a prospective study. $J$ Pediatr 1974:85:848-52.

- Simpson H. Muk JYQ. Outcome of respiratory disease in childhood. In: Milner AD. Martin RJ, eds. Neonatal and paediatric respiratory medicine. London: Butterworths, 1986: 211-35.

${ }^{3}$ Samet JM. Tager IB. Speizer FE. The relationship between respiratory illness in childhood and chronic airflow obstruction in adulthood. Am Rev Respir Dis 1983:127:508-23.

4 Ferris BG. Epidemiology Standardization Project: Recommended respiratory disease questionnaire for use with adults and children in epidemiological research. Am Rev Respir Dis 1978:118(suppl 7):36-53.

5 Harrald FJC. McMichael J. Determination of lung volume: simple constant volume modification of Christic's method. Proc $R$ Soc London 1939;126B:491.

- Polgar G. Promadhat V. Pulmonary function testing in children. techniques and standards. Philadelphia: WB Saunders, 1971.

7 Knudson RJ, Lebowitz MD. Holberg CJ. Burrows B. Changes in the normal maximal expiratory flow-volume curve with growth and agcing. Am Rev Respir Dis 1983;127:725-34.

* Silverman M. Anderson SD. Standardization of exercise tests in asthmatic children. Arch Dis Child 1973:47:882-9.

"Lee DA. Winslow NR. Speight ANP. Hey EN. Prevalence and spectrum of asthma in childhood. Br Med J 1983;286:1256-8.

16) Andersen HR, Bailey PA, Cooper JS, Palmer JC, West S. Medical care of asthma and wheezing illness in children: a community survey. J Epidemiol Community Health 1983;37: $180-6$.

" Gurwitz D, Kattan M, Levison H, Culham JAG. Pulmonary function abnormalities in asymptomatic children after hydrocarbon pncumonitis. Pediatrics 1978;62:789-94.

12 Laughin JJ, Eigen H. Pulmonary function abnormalities in survivors of near drowning. J Pediatr 1982;100:26-30.

1.3 Mok JYQ, Simpson H. Outcome of acute bronchitis, bronchiolitis and pncumonia in infancy. Arch Dis Child 1984:59:306-9.

Correspondence and requests for reprints to Dr PI Macfarlane. Respiratory Unit, Royal Liverpool Children`s Hospital Alder Hey, Liverpool L12 2AP. England.

Accepted 6 November 1987 